\title{
On Distributed Computation in Noisy Random Planar Networks
}

\author{
Yashodhan Kanoria and D. Manjunath \\ Department of Electrical Engineering \\ Indian Institute of Technology Bombay \\ Mumbai 400076 INDIA \\ yashodhan, dmanjulee.iitb.ac.in
}

\begin{abstract}
THIS PAPER IS ELIGIBLE FOR THE STUDENT PAPER AWARD. We consider distributed computation of functions of distributed data in random planar networks with noisy wireless links. We present a new algorithm for computation of the maximum value which is order optimal in the number of transmissions and computation time. We also adapt the histogram computation algorithm of Ying et al [1] to make the histogram computation time optimal.
\end{abstract}

\section{INTRODUCTION}

We consider distributed or 'in-network' computation of functions of sensing data in sensor networks in two dimensions. The sensor nodes collect sensing data and communicate with other nodes in a limited range over noisy wireless links. Our interest is in efficient evaluation of specific functions of sensing data. Latency, energy cost and throughput are the efficiency measures. We assume that communication costs dominate the time and energy cost for the computations.

Two types of wireless networks are considered in the literature studying function computation-random planar networks [1]-[3] and collocated or broadcast networks [2], [4][6]. Results for collocated networks are useful in solving corresponding problems for random planar networks. While [2], [3] consider noise-free links, [1], [4]-[6] consider noisy links. Computation of the histogram, and hence that of any symmetric function, in a random planar network with noisy links is considered in [1]. A protocol requiring $\Theta(n \log \log n)$ transmissions to compute the histogram under the strong noise model is described. The lower bound is open. [1] is the only study of distributed computation in a noisy random planar network and it does not obtain the computation time (which is not the same as the energy for a random planar network). In this paper, we show how to effectively use the coding theorem of [7] to reduce the computation time in noisy random planar networks for maximum (MAX) and histogram functions. Also, our protocol for MAX requires $\Theta(n)$ transmissions.

\section{Model And PRoblem Description}

Sensor nodes $1,2, \ldots, n$ are uniformly distributed in $[0,1]^{2}$. The transmission range of all the nodes is $r_{n}$. We use the protocol model of interference [8]. Let $\rho_{i, j}$ be the distance between nodes $i$ and $j$. A transmission of node $i$ can be received at node $j$ if $\rho_{i, j} \leq r_{n}$, and for every other simultaneously transmitting node $k, \rho_{k, j} \geq(1+\Delta) r_{n}$, for some constant $\Delta$.
If $\rho_{i, j}<r_{n}$ and there exists a node $k$ that is transmitting at the same time as node $i$ such that $\rho_{k, j} \leq(1+\Delta) r_{n}$, then there is a collision at node $j$.

Time is slotted and all nodes are synchronized to these slots. The slot duration is equal to the bit duration. At time $t$, sensor node $i$ has data $x_{i}(t)$ with $x_{i}(t)$ taking values from a finite set. For simplicity, we assume $x_{i}(t)$ is $\{0,1\}$-valued. (A general finite set only changes the results by a constant factor.) Define $x(t):=\left[x_{1}(t), x_{2}(t), \ldots, x_{n}(t)\right]$. Our interest is in efficiently computing $f(t):=\phi(x(t))$ at some instants of time. $f(t)$ is to be made available at a single sink node $s$, which is a node close to the center of the unit square. In general, $f(t)$ may be required at a subset of the nodes.

There are many ways in which the computation of $f(t)$ for different $t$ can be scheduled. Three such computation models are usually discussed in the literature. (1) One shot computation is a one-time computation of $f(\cdot)$ and, without loss of generality, we consider only $f(0)$. (2) In pipelined computation $f(t)$ is to be computed for $\left\{t_{k}\right\}_{k=1,2, \ldots}$ with the computations for the different $t_{k}$ being pipelined at each of the nodes. (3) In block computation $x(t)$ is collected for a block of, say, $K$ instants, i.e., for $\left\{t_{k}\right\}_{k=1, \ldots, K}$ and $f(t)$ is computed for this block of time.

The wireless links in the network are assumed to be binary symmetric channels with errors independent across receivers. We assume that the error probability is upper bounded by some $\epsilon_{0}<0.5$. Thus, our protocols work for the strong clairvoyant adversary model [9]. Note that the protocols in [1], [4], [5] also work for this noise model. A weaker noise model where each bit is in error with probability exactly $\epsilon_{0}$ has also been considered in the literature, e.g., [10].

Different distributed scheduling strategies are possible. In a collision free strategy (CFS), transmissions are scheduled such that in the absence of noise, there is no collision at the intended receivers. Also, information cannot be communicated by a node by avoiding a transmission in its scheduled time slot. This means that even though our model for the noisy network does not allow bit erasures, the protocols designed are robust in their presence. Erasures, if they occur, can be immediately identified as errors for such protocols. A CFS may be oblivious or non-oblivious. In an oblivious protocol, the transmission schedule is fixed beforehand. In a non-oblivious protocol, the data values transmitted in previous slots determine the 
evolution of the schedule. A non-oblivious CFS designed for a noiseless network can result in collisions when used in a noisy network. An oblivious protocol ensures that such collisions do not occur. We look for oblivious protocols.

Let $E_{t}$ be the energy consumed for transmission of one bit, $E_{r}$ be the energy consumption at the receiver of a transmission. We ignore processing energy. In general, $E_{t}$ depends on $r_{n}$, and hence on $n$. However, results in [3] indicate that for sufficiently large $n(\geq 5000)$, it is reasonable to assume that the transmission energy is constant and independent of $n$. We follow this assumption. Note that this is different from the energy model of [1]. If we let $E_{r}<<E_{t}$, the number of transmissions required in the protocol can be used as a proxy for the energy consumed. Energy model $E_{1}$ will refer to the case when $E_{r}$ is not to be ignored and $\mathrm{EM}_{2}$ will refer to the case when it can be ignored. In $\mathrm{EM}_{2}$, we simply count the number of transmissions. Multiplying the energy in $\mathrm{EM}_{2}$ by $k_{5}\left(\sqrt{\frac{\log n}{n}}\right)^{\alpha}$ for some constant $k_{5}$ and $\alpha$ gives the energy in the model of [1].

As was mentioned earlier, we will consider the evaluation of the maximum (MAX) and histogram of $x(t)$. Note that the MAX is the same as the OR function for binary data.

\section{A. Summary of Results and Organization of the Paper}

We describe a single oblivious protocol for computation of MAX in a noisy network that matches the lower bounds for both time and transmissions of the optimal CFS in a noise free network. This may be compared against the $\log \log n$ penalty in number of transmissions that seems inevitable for the histogram function [1]. We also show how to achieve the trivial lower bound in time for histogram computation in a noisy random planar network, using a suitable modification of the algorithm presented in [1]. The number of transmissions is the same as that in [1], i.e., $\Theta(n \log \log n)$.

\section{MAX IN A NoISy Random Planar NeTwork}

Like in [3], we wish to evaluate $f=$ MAX/OR. Following [1]-[3], we propose a two-stage algorithm which is implemented by dividing the operational area into appropriately sized cells, performing intra-cell computation, or fusion, and then propagating the fused results towards the sink with appropriate combination on the way.

Following [11], we perform a tessellation of the unit square into square cells of side $l=\left[\sqrt{\frac{n}{2.75 \log n}}\right]^{-1}$. This gives us a total of $M_{n}=\left[\sqrt{\frac{n}{2.75 \log n}}\right]^{2}$. Label the cells as $S_{j}, j=$ $1,2, \ldots, M_{n}$, from left to right and from top to bottom as shown in Fig. 1. Let $N_{j}$ denote the number of sensor nodes in cell $S_{j}$. Of course $\sum_{j=1}^{M_{n}} N_{j}=n$. Choose $r_{n}=\sqrt{\frac{13.75 \log n}{n}} \approx$ $\sqrt{5}$ l. Since $l \leq \frac{r_{n}}{\sqrt{2}}$ the nodes in each of the cells form a single hop network. Lemma 3.1 of [11] provides us with useful bounds on the number of nodes in each cell.

Lemma 1: For any $\mu>\mu^{*}=0.9669$,

$$
\lim _{n \rightarrow \infty} \operatorname{Pr}\left(\max _{j}\left|N_{j}-2.75 \log n\right| \leq 2.75 \mu \log n\right)=1
$$

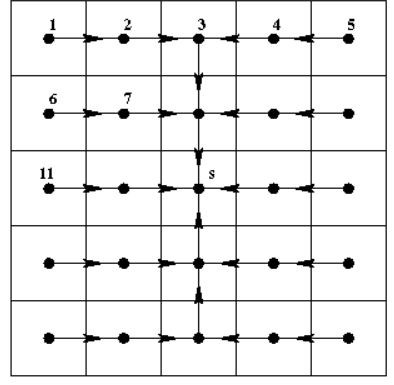

Fig. 1. The tessellation of the unit square with labeling of cells. The spanning tree used in inter-cell communication is also shown.

The above lemma means that for $j=1,2, \ldots, M_{n}$, with probability 1 , as $n \rightarrow \infty$

$$
0.091 \log n \leq N_{j} \leq 5.41 \log n
$$

We form a spanning tree of the cells rooted at cell $S_{s}$ with edges along the central vertical axis and along all horizontal rows of cells as shown in Fig. 1. Let $\pi(j)$ denote the index of the parent of cell $j$, i.e. cell $S_{\pi(j)}$ is the parent of $S_{j}$, $j=1,2, \ldots, s-1, s+1, \ldots, M_{n}$. We arbitrarily select one node in each cell $S_{j}$ to be the cell center $C_{j}$ (except that the sink node is made the cell center of $S_{s}$ ). This is possible with high probability since (2) tells us that each cell is occupied. The cell centers of two adjacent cells are one-hop neighbors, since $l \leq \frac{r_{n}}{\sqrt{5}}$.

\section{A. Stage 1: Intra-cell Computation}

At the end of the first stage, the maximum of the values in each cell $S_{j}$ is to be made available to $C_{j}$. Since each cell behaves like a single-hop network, we can use a noisy broadcast network protocol for this purpose.

It helps to review the protocol for a noiseless random planar network [3]. Each node transmits its bit to the cell center. From (2), it is sufficient to give each cell $\lfloor 5.41 \log n\rfloor$ time slots. During the computation in each cell, at most $k_{1}:=$ $\left(2\left\lceil\frac{(1+\Delta) r_{n}}{l}\right\rceil+1\right)^{2}-1$, i.e., a constant number of adjacent cells will be disabled due to interference. Thus, with high probability, this stage will require at most $\left(k_{1}+1\right)\lfloor 5.41 \log n\rfloor$ slots, i.e., $\Theta(\log n)$ time using $\Theta(n)$ total transmissions.

For a noisy network, [1] describes a protocol using which the aggregation in a cell can be performed in $\Theta(\log n \log \log n)$ slots (with scheduling of cells as above) using $\Theta(n \log \log n)$ total transmissions with overall probability of error $O\left(\frac{1}{\log n}\right)$. Using this intra-cell protocol, we can collect the histogram of the cell sensor readings at the cell-center. However, this is more information than we need. Instead, we adapt the algorithm of [5] to reduce the complexity of the intra-cell protocol to $O(n)$ transmissions. We explain this adaptation next.

Define the 'witness' for cell $S_{j}, \hat{W}_{j}$, as follows. $\hat{W}_{j}=i_{0}$ if $i_{0}$ is the minimum $i$ for which $x_{i}=1$ and $i \in S_{j}$. If $x_{i}=0$ for all $i \in S_{j}$, an arbitrary node can be designated as $\hat{W}_{j}$. 
Each cell is a single-hop network and we run the "witness discovery' protocol described in the proof of Theorem 2 of [5]. From Theorem 2 of [5], witness discovery in $S_{j}$ can be achieved by an oblivious protocol with less than $k_{2} N_{j}$ bit-transmissions in $S_{j}$, for some constant $k_{2}$. At the end of the execution of this protocol, $C_{j}$ identifies some $W_{j}$ as the witness for $S_{j}$ such that $\operatorname{Pr}\left(W_{j} \neq \hat{W}_{j}\right)<\epsilon_{1}$ for any desired constant $\epsilon_{1}>0$. We can therefore achieve $\operatorname{Pr}\left(x_{W_{j}} \neq \max _{i_{i} i \in S_{j}}\left\{x_{i}\right\}\right)<\epsilon_{1}$, for any $\epsilon_{1}>0$ i.e., $W_{j}$ has the correct value of the maximum of the values in cell $j$ with error probability that can be upper bounded by any constant. If $\epsilon$ is the desired bound for the error probability in computing $f$, we choose $\epsilon_{1}=\frac{\varepsilon}{2}$ for each cell.

From Observation 2.1 in [5], $C_{j}$ can distribute the identity of $W_{j}$ to all nodes in $S_{j}$ using $\Theta(\log n)$ transmissions to ensure that the identity of $W_{j}$ is known to all nodes in the cell with error probability $O\left(\frac{1}{n}\right)$. Finally, $W_{j}$ transmits $x_{W_{j}}, \Theta(\log n)$ times to $C_{j}$ to ensure that the probability of $C_{j}$ having the wrong value of $x_{W_{j}}$ is $O\left(\frac{1}{n}\right)$. Let $f_{j}$ denote the value of $x_{W_{j}}$ decoded by $C_{j}$ from these transmissions. Note the use of $\Theta(\log n)$ transmissions and not $\Theta\left(\log N_{j}\right)$ transmissions in the last two steps to ensure error probability is $O\left(\frac{1}{n}\right)$ for each of the cells.

Thus, $\Theta(\log n)+k_{2} N_{j}$ transmissions suffice to complete Stage 1 in cell $S_{j}$. Using (2) we can find constants $k_{3}$ and $k_{4}$ such that Stage 1 requires $a_{j} N_{j}=\Theta(\log n)+k_{2} N_{j}$ transmissions in $S_{j}$ with $k_{3}<a_{j}<k_{4} \forall j=1,2, \ldots, M_{n}$. This, along with the bound on the number of interfering neighbors for a cell, means that Stage 1 can be completed in the entire network in $\Theta(\log n)$ time using $\Theta(n)$ transmissions.

While the probability of error in correctly identifying $W_{j}$ in each cell $j$ is upper bounded by a constant, we do not yet know a 'network-wide' error probability. But then how do we define the error in the network? An obvious definition of a 'networkerror' is the event of there being an error in identifying the witness in one or more cells. A little consideration shows that a more appropriate definition of the 'error' would be as follows. Let $f:=\max _{1 \leq i \leq n}\left\{x_{i}\right\}$ be the true value of the MAX function, and let $\hat{f}:=\max _{1 \leq j \leq M_{n}}\left\{f_{j}\right\}$. We will say that $\gamma_{1}$, a Stage 1 error, has occurred, if $f \neq \hat{f}$. We show that the probability of $\gamma_{1}$ is bounded by a constant for large $n$.

An error in stage 1 (as defined above) occurs in two ways,

1) $f=1$ and $f_{j}=0 \forall j$. In this case, there must be a cell $S_{\hat{j}}$ that has at least one 1 . Now the probability of missing all $x_{i}=1$ for $i \in S_{\hat{j}}$ in identifying the witness for cell $S_{\hat{j}}$ is bounded by $\frac{\varepsilon}{2}$. The probability of finding a witness $W_{\hat{j}}$ with $x_{W_{\hat{3}}}=1$ but having $f_{\hat{j}}=0$ is $O\left(\frac{1}{n}\right)$. Thus the probability of having $f_{\hat{\jmath}}=0$ is bounded by $\frac{\varepsilon}{2}+O\left(\frac{1}{n}\right)$. This gives us a bound of $\frac{\varepsilon}{2}+O\left(\frac{1}{n}\right)$ on the probability of the event $\gamma_{1}$.

2) $f=0$ and $f_{j}=1$ for one or more $j$. Since $f=0$, we have $x_{W_{j}}=0 \forall j$. The probability of obtaining the wrong value of $f_{j}$ is $O\left(\frac{1}{n}\right)$ for $1 \leq j \leq M_{n}$. Since there $M_{n}=\Theta\left(\frac{n}{\log n}\right)$ cells, from the union bound, the probability of $\gamma_{1}$ is $O\left(\frac{1}{\log n}\right)$.

Thus the error probability for the Stage 1 is bounded by $\frac{\varepsilon}{2}+$ $O\left(\frac{1}{\log n}\right)$.

Using the proof technique of Theorem 4 of [5], an oblivious protocol can be designed to achieve the same asymptotic time and transmissions complexity as the query based protocol. The initial phase in which each node transmits its data value a constant number of times and is heard by all other nodes in its cell requires $\Theta(n \log n)$ energy under $\mathrm{EM}_{1}$. We already know that the energy is $O(n \log n)$ under $\mathrm{EM}_{1}$, since each transmission can be heard by at most $\Theta(\log n)$ nodes. Hence, the total energy consumption of the first stage is $\Theta(n \log n)$ under $E M_{1}$. Because of the phase in which the identity of $W_{j}$ is distributed, this is also the energy consumption for the non-oblivious version described earlier.

Lemma 2: Stage 1 can be completed by an oblivious protocol in time $\Theta(\log n)$ using $\Theta(n)$ transmissions (same as energy under $\left.\mathrm{EM}_{2}\right)$ with energy consumption $\Theta(n \log n)$ under $\mathrm{EM}_{1}$, such that the overall probability of error is bounded by $\frac{\varepsilon}{2}+O\left(\frac{1}{\log n}\right)$.

\section{B. Stage 2: Inter-cell Computation}

As in [1]-[3], in Stage 2, data is passed along the tree towards the sink, first horizontally and then vertically between the cell centers $C_{j}$ (see Fig. 1). Let $\hat{f}_{j}=\max _{j^{\prime}}\left\{f_{j^{\prime}}\right\}$ where $S_{j^{\prime}}$ are the cells in the subtree rooted at cell $S_{j}$, excluding cell $S_{j} . \max \left\{f_{j}, \hat{f}_{j}\right\}$ is passed to the cell center of the parent cell $C_{\pi(j)}$. Data thus moves along the spanning tree towards the sink.

In this stage, parallel communication of data can reduce the time complexity. Recall that when $C_{j}$ transmits, at most $k_{1}$ other adjacent cell centers will not be able to receive other transmissions in the same slot. Note that we need bidirectional flow of data along the edges of the tree to use [7]. Since the vertices of the tree have a maximum degree of $d=4$, for an active link, at most $k_{5}=4\left(k_{1}+1\right)$ links incident on the cells in the neighborhood will be disabled. Thus, all links can be activated at least once each in at most $k_{5}$ slots. We can therefore consider each link in the spanning tree to be a 1-bit per 'logical slot' link with each logical slot corresponding to $k_{5}$ real slots. Hereafter, we consider only logical time slots.

It is easy to see that the coding theorem of [7] is applicable to the logical network defined above.

Theorem 1: (Theorem 1.1 of [7]) Any protocol which runs in time $T$ on an $N$-processor network of degree $d$ having noiseless communication channels, can, if the channels are noisy (each a binary symmetric channel of capacity $C, 0<C \leq 1$ ), be simulated on that network in time $O\left(T \frac{1}{C} \log (d \overline{+}+1)+\frac{1}{C} \log N\right)$ with probability of error $e^{-\Omega(T)}$.

Remark 1: The protocol described in [7] to achieve this uses tree codes. [12] gives the best available construction of tree codes-a randomized construction that works correctly with probability arbitrarily close to 1 . The computation required for maximum likelihood decoding used in the proof of 


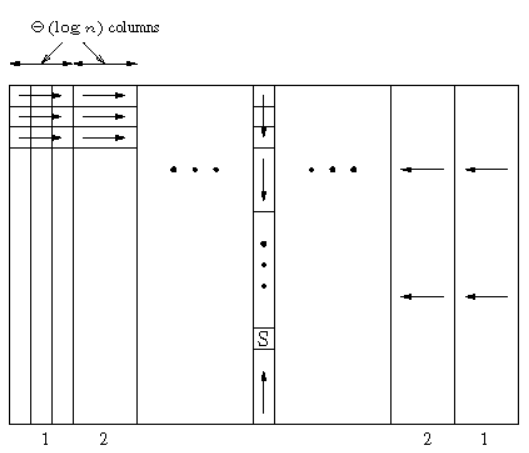

Fig. 2. Sub-stages in the Inter-cell protocol. Part of the unit square is shown. $S$ denotes the cell containing the sink.

Theorem 1 grows exponentially with the depth of the tree and an efficient method for decoding still needs to be found to make it computationally tractable. We ignore computational expense in this paper.

The depth of the tree is $\Theta\left(\sqrt{\frac{n}{\log n}}\right)$ and there are $\Theta\left(\frac{n}{\log n}\right)$ nodes in the tree. Hence Stage 2 would require $\Theta\left(\sqrt{\frac{n}{\log n}}\right)$ time slots and $\Theta\left(\frac{n}{\log n}\right)$ transmissions in a noiseless network. We can modify this protocol as follows to make it possible to use Theorem 1 to simulate it in a noisy network-for each time slot in which a link is not activated send a dummy bit on the link (say 0). Now, from Theorem 1, this protocol can be simulated in the noisy network with only constant factor increase in time because we have $d=4$. However, a naive implementation of the protocol of [7] would need $\Theta\left(\left(\frac{n}{\log n}\right)^{\frac{3}{2}}\right)$ transmissions, compared to $\Theta\left(\frac{n}{\log n}\right)$ transmissions for the noiseless network. There is an increase of $\Theta\left(\sqrt{\frac{n}{\log n}}\right)$, i.e., $\Theta\left(\frac{1}{r_{n}}\right)$, because the protocol of [7] requires every $C_{j}$ to transmit in every slot, while in the noiseless network, each would transmit just once.

We can reduce the number of transmissions by securing the transmissions at each level of the tree by repetition coding, i.e., transmitting $\Theta(\log n)$ times on each link. This increases the number of transmissions to $\Theta(n)$, an increase of only $\Theta(\log n)$ from the noiseless case. Since each node has to collect the data from the tree below it, we can use information theoretic arguments to show that even if we interleave transmissions at different depths of the tree in time, we cannot do better than this in terms of number of transmissions. Note that the overall number of transmissions for the maximum computation is now just $\Theta(n)$ i.e., it is optimal. However, there is a penalty of $\Theta(\log n)$ in time, which is inflated to $\Theta(\sqrt{n \log n})$.

Ideally, we would like a way to complete the Stage 2 computation in $O(n)$ transmissions (at most as many transmissions as Stage 1) and in $\Theta\left(\sqrt{\frac{n}{\log n}}\right)$ time i.e., we would like to not have a penalty either in time or in number of transmissions. Surprisingly, this is possible.

This can be achieved by propagation of bits up the tree in a series of sub-stages, moving $\Theta(\log n)$ levels (ignoring edge effects) in $\Theta(\log n)$ time in each sub-stage. The first set of sub-stages bring data to the central axis, and the second set take it to the sink. There are $\Theta\left(\sqrt{\frac{n}{(\log n)^{3}}}\right)$ sub-stages. See Fig. 2.1 and 2 in the figure correspond to sub-stages.

At each sub-stage we have $O\left(\frac{1}{r_{n}}\right)$ linear array networks with $\Theta(\log n)$ nodes each. From Theorem 1 , we can ensure that the error probability is $O\left(\frac{1}{n}\right)$ for each linear array. This gives a bound of $O\left(\sqrt{\frac{1}{n \log n}}\right)$ for the sub-stage error probability. We have an overall union bound of $O\left(\frac{1}{(\log n)^{2}}\right)$ for the error probability of Stage 2. Note that a vanishing error probability would be impossible if each sub-stage involved $o(\log n)$ levels of the tree in an attempt to reduce the number of transmissions without increasing the time. Clearly, each of the $\Theta\left(\frac{n}{\log n}\right)$ links is activated $\Theta(\log n)$ times in this scheme i.e., $\Theta(n)$ transmissions are required in total. Each transmission is to be heard by a single receiver, hence the energy under both $\mathrm{EM}_{1}$ and $\mathrm{EM}_{2}$ is $\Theta(n)$.

Lemma 3: Stage 2 can be completed by an oblivious protocol in $\Theta\left(\sqrt{\frac{n}{\log n}}\right)$ time using $\Theta(n)$ transmissions (same as energy under $E M_{2}$ ) with energy consumption $\Theta(n)$ under $E M_{1}$, such that the overall probability of error is bounded by $O\left(\frac{1}{(\log n)^{2}}\right)$.

The total error for the computation is $\frac{\varepsilon}{2}+O\left(\frac{1}{\log n}\right)+$ $O\left(\frac{1}{(\log n)^{2}}\right)=\frac{\varepsilon}{2}+O\left(\frac{1}{\log n}\right)<\epsilon$ for large $n$.

Combining lemmas 2 and 3 :

Theorem 2: In a random planar network with binary data, with binary symmetric channels and errors independent across receivers with error probability bounded by some $\epsilon_{0}<\frac{1}{2}$, with high probability the MAX (or $O R$ ) can be computed with probability of error less than any $\epsilon>0$ for large $n$ using $\Theta(n)$ total transmissions with transmission radius $\Theta\left(\sqrt{\frac{\log n}{n}}\right)$ and in a time $\Theta\left(\sqrt{\frac{n}{\log n}}\right)$.

This matches the corresponding results for noiseless networks in [3]. The total energy for the protocol described is $\Theta(n \log n)$ under $\mathrm{EM}_{1}$ and $\Theta(n)$ under $\mathrm{EM}_{2}$.

\section{Discussion}

\section{A. Pipelining}

It is easy to see that a pipelined throughput of $\Theta\left(\frac{1}{\log n}\right)$ with a delay of $\Theta\left(\sqrt{\frac{n}{\log n}}\right)$ in reaching the sink is also achievable in the noisy case with a scheme similar to the one given in [3] for the noiseless case. We alternately perform iterations of Stage 1 and Stage 2, spending $\Theta(\log n)$ time on each. While doing Stage 2, we allow for one sub-stage of each of the ongoing computations to be completed. Note that different sub-stages of different computations can essentially be done in parallel. 


\section{B. Distribution of computation result}

The result of the MAX computation can be distributed to all nodes with only a constant factor overhead in time and number of transmissions. It can be distributed to the cell centers along the same spanning tree in a series of sub-stages as in Stage 2 (Section III-B). Thereafter, each cell center can broadcast the value $\Theta(\log n)$ times so that all nodes have the correct value of the function with error probability $O\left(\frac{1}{n}\right)$.

\section{Non-binary data}

All results can easily be generalized to the case in which sensor data belongs to any finite set. Suppose $x_{i} \in \mathcal{X}=$ $\{1,2, \ldots,|\mathcal{X}|\} \quad \forall i=1,2, \ldots, n$. We can complete the computation of MAX in $\log _{2}|\mathcal{X}|$ stages. Each stage consists of two parts. Briefly, the first part is as described in Section III, and reduces the number of possibilities for the result by a factor of 2 . The second part involves distribution of the result of the first part as in Section IV-B. Thus the computation can be completed using $\Theta(n \log |\mathcal{X}|)$ total transmissions in a time $\Theta\left(\sqrt{\frac{n}{\log n}} \log |\mathcal{X}|\right)$.

\section{Block Computation}

The results of Giridhar and Kumar [2] carry over trivially to the case of noisy networks. This is because in block computation, Gallager's coding theorem can be used to secure each individual message transmission with a constant factor overhead, provided the message length is long enough. This can be ensured simply by taking sufficiently long blocks. MAX belongs to the class of type-threshold functions and can hence be computed at a rate of $\Theta\left(\frac{1}{\log \log n}\right)$.

\section{OPTIMIZING THE TIME OF HISTOGRAM COMPUTATION}

The protocol for histogram computation with binary data in a noisy random planar network in [1] takes $\Theta(n \log \log n)$ transmissions. Computation time is ignored. Clearly, the time required by any correct protocol for histogram computation must be $\Omega\left(\sqrt{\frac{n}{\log n}}\right)$ (as is true even for MAX). We describe an algorithm that takes $\Theta\left(\sqrt{\frac{n}{\log n}}\right)$ time slots.

Firstly, we construct a spanning tree as in Fig. 1. This ensures that the depth of the tree is limited to $\Theta\left(\sqrt{\frac{n}{\log n}}\right)$. This is in contrast with an arbitrarily constructed spanning tree which can have a depth as large as $\Theta\left(\frac{n}{\log n}\right)$. Even with the spanning tree of Fig. 1, an implementation of the Inter-cell protocol described in [1] requires $\Theta(\log n)$ time for each level of the tree, yielding a total time requirement of $\Theta(\sqrt{n \log n})$. Number of transmissions needed is $\Theta(n)$. We show how to bring the time required down to $\Theta\left(\sqrt{\frac{n}{\log n}}\right)$ while using the same number of transmissions.

Once again we perform Stage 2 in a series of sub-stages as shown in Fig. 2. Each cell needs to convey the number of 1's in its sub-tree of cells (including the cell itself) to its parent. This information can be represented by a binary string of size $g=\lceil\log n\rceil$ for each cell (by adding appropriate zeros to the left). Let this binary string for cell $S_{j}$ be $b_{g-1}^{j} b_{g-2}^{j} \ldots b_{0}^{j}$, where each $b_{k}^{j}$ is a bit. Consider part of a sub-stage with the linear array of cells $S_{a_{1}}, S_{a_{2}}, \ldots, S_{a_{q}}$, where $q=\Theta(\log n)$ (ignoring edge effects). The cells are ordered with $S_{a_{0}}$ being the cell at the greatest depth, and $S_{a_{q}}$ being the cell closest to the sink. The noiseless protocol to be simulated using Theorem 1 is constructed as follows. $C_{a_{1}}$ transmits $b_{0}^{a_{1}}$ up to the cell center of its parent $C_{a_{2}}$ in the first time slot. Other links have dummy bits exchanged. Before the second slot, $C_{a_{2}}$ computes $b_{0}^{a_{2}}$ using the number of 1 's in cell $S_{a_{2}}$ and the received bit $b_{0}^{a_{1}}$. In the second slot, $C_{a_{1}}$ sends $b_{1}^{a_{1}}$ to $C_{a_{2}}$, and $C_{a_{2}}$ sends $b_{0}^{a_{2}}$ to $C_{a_{3}}$. Dummy bits are sent over other links. Before the third slot, $C_{a_{2}}$ computes $b_{1}^{a_{2}}$ and $C_{a_{3}}$ computes $b_{0}^{a_{3}}$. Progressing this way, at the end of $q+g-1=$ $\Theta(\log n)$ slots, $C_{a_{q}}$ has received all required data from $C_{a_{q-1}}$. Now, this protocol can be simulated in a noisy setting using Theorem 1. The sub-stage can be completed in $\Theta(\log n)$ time, using $\Theta(\log n)$ transmissions for each cell center (as for the MAX computation). Moreover, we can ensure that the overall probability of error goes to zero as for the MAX computation. Thus, the inter-cell protocol can be completed in $\Theta\left(\sqrt{\frac{n}{\log n}}\right)$ time overall, using only $\Theta(n)$ transmissions.

\section{CONCLUSION}

A non-trivial lower bound is yet to be proved for number of transmissions required for histogram computation. [6] demonstrates that $\Theta(n)$ transmissions suffice in a noisy broadcast network (in a weak noise model). However, for a noisy random planar network, the best known algorithm requires $\Theta(n \log \log n)$ transmissions, whereas the trivial lower bound is $\Omega(n)$. It would be interesting to close the gap.

Also note that the computational complexity of the algorithms that we describe here can be significant, given that an efficient method for decoding of tree codes is not known.

\section{REFERENCES}

[1] L. Ying, R. Srikant, and G. Dullerud, "Distributed symmetric function computation in noisy wireless sensor networks with binary data", in Proc. WiOpt, Apr 2006.

[2] A. Giridhar and P. R. Kumar, "Computing and communicating functions over sensor networks", IEEE Jour on Sel. Areas in Commun., vol. 23, no. 4, pp. 755-764, Apr 2005.

[3] N. Khude, A. Kumar, and A. Karnik, "Time and energy complexity of distributed computation in wireless sensor networks", in Proc. IEEE INFOCOM, 2005, vol. 4, pp. 2625-2637.

[4] R. G. Gallager, "Finding parity in simple broadcast networks", IEEE Trans. on Info. Theory, vol. 34, pp. 176-180, 1988.

[5] I. Newman, "Computing in fault tolerance broadcast networks", in 19 th IEEE Conf. on Computational Complexity, 2004, pp. 113-122.

[6] N. Goyal, G. Kindler, and M. E. Saks, "Lower bound for the noisy broadcast problem.", in FOCS, Oct. 2005, pp. 40-52.

[7] S. Rajagopalan and L. J. Schulman, "A coding theorem for distributed computation", in Proc. 26th STOC, 1994, pp. 790-799.

[8] P. Gupta and P. R. Kumar, "The capacity of wireless networks", IEEE Trans. On Info. Theory, vol. 46, no. 2, pp. 388-404, Mar 2000.

[9] U. Feige and J. Kilian, "Finding or in noisy broadcast network", Information Processing Letters, vol. 73, no. 1-2, pp. 69-75, Jan 2000.

[10] E. Kushilevitz and Y. Mansour, "Computation in noisy radio networks", in Proc. SODA, 1998, pp. 236-243.

[11] F. Xue and P. Kumar, "The number of neighbors needed for connectivity of wireless networks", Wireless Networks, vol. 10, no. 2, pp. 169-181, Mar 2004.

[12] M. Peczarski, "Strategy in Ulam's game and tree code give errorresistant protocols", Arxiv preprint, cs.DC/0410043, 2004. 